\title{
Neuropsycological Function, Insight, and Treatment Adherence among Patients with Schizophrenia
}

\author{
Deroui $\mathrm{K}^{* 1}$, Bouslikhane $\mathrm{M}^{1}$, Abouelfaraj $\mathrm{H}^{1}$, Malki $\mathrm{Z}^{1}$, Agoub $\mathrm{M}^{1,2}$ and Battas $\mathrm{O}^{1,2}$ \\ ${ }^{1}$ Departement of Psychiatry,Ibn Ruschd University, Casablanca, Morocco \\ ${ }^{2}$ Clinical Neuroscience and Mental Health Laboratory, University Hassan II, Casablanca, Morocco
}

${ }^{*}$ Corresponding author: Deroui K, Departement of Psychiatry,Ibn Ruschd University, Casablanca, Morocco, E-mail: khadijaderoui123456789@gmail.com

Citation: Deroui K, Bouslikhane M, Abouelfaraj H, Malki Z, Agoub M, et al. (2017) Neuropsycological Function, Insight, and Treatment Adherence among Patients with Schizophrenia. J Cas Rep Stud 5(6): 605

Received Date: May 06, 2017 Accepted Date: December 27, 2017 Published Date: December 29, 2017

\begin{abstract}
Introduction: Research has suggested that poor insight in patients with schizophrenia is associated with poorer treatment compliance. One factor ontributing to poor insight may be neurocognitive deficits.

Objectives: The aim of the current study is to explore the relationship between cognitive insight, clinical insight, treatment adherence, executive functions and sustained attention within patients with schizophrenia.

Methods: The study was conducted on 41 patients with schizophrenia using the Beck cognitive insight scale, the schedule for assessment of insight, the drug attitude inventory and two neuropsychological tests.

Results: A correlation was found between the clinical insight and the treatment compliance, but no correlation was found between clinical insight and neuropsychological tests.

Conclusion: This study suggests that poor insight is among factors of poor compliance to treatment in schizophrenia but doesn't support the hypothesis that cognitive impairment may underlie poor insight in schizophrenia.

Keywords: Neuropsychological function; Insight; Treatment adherence; Schizophrenia
\end{abstract}

\section{Introduction}

Initially, insight was considered a dichotomous construct that was either present or absent within patients with schizophrenia, and was measured by a patient's verbal recognition of existing psychological difficulties. This conceptualization, however, has evolved into one of multidimensionality, where insight incorporates a multitude of phenomena [1].

David (1990) suggested that insight is composed of 3 distinct overlapping dimensions: recognition that one has a mental illness, compliance with the treatments and ability to recognize psychotic events (delusions and hallucinations) as pathological [2].

The importance of maintenance therapy in schizophrenia has been well established, the risk of relapse increases almost 5-fold when antipsychotic drug therapy is discontinued. Nonadherence to medication worsens symptoms and increases suicidal attempts and, consequently, emergency room visits or re-hospitalization. Understanding the determinants of nonadherence in schizophrenia is thus important for developing effective relapse prevention strategies [3]; and lack of insight is frequently cited as a significant factor of medication nonadherence [1].

One factor contributing to impaired insight in psychotic disorders may be neucognitive deficits [1].

Broadly speaking, researches had emphasized that poor insight is significantly related with executive functioning [2]. Executive skills permit an adaptive balance of maintenance and shifting of cognitive or behavioral responses to the environmental demands, permitting longer-term goal-directed behavior [2].

If lack of insight is underpinned by cognitive impairment, then it may require a therapeutic approach which is different from that currently afford; however, several studies have yielded contradictory results concerning the role of cognition, and insight, raising doubts about their predictive power [4].

Hence the confusion regarding the impact of executive function on insight still exists. In Morocco, there is a prominent lack of literature addressing this issue. 


\section{Objectives}

The aim of this current study is to explore the relationship between cognitive insight, clinical insight, treatment adherence, executive functions and sustained attention within patients with schizophrenia.

\section{Materials and Methods}

\section{Site of study}

The study was conducted in July/ September 2013 in the University Psychiatric Center Ibn Rushd of Casablanca.

\section{Population studied}

The study was conducted among forty one inpatients diagnosed with schizophrenia according to the DSM-IV criteria. An informed consent was requested from patients being insured by anonymity of collected data.

\section{Methods}

The following data were collected: Age, gender, and educational level, age of the onset of the disorder, substance misuse, number of hospitalizations and drug information.

\section{Measures}

BECK Cognitive Insight Scale (BCIS): This scale was developed to evaluate patients' self-reflectiveness and their overconfidence in their interpretations of their experiences. It's a 15-item self-report questionnaire yielding a 9-item self-reflectiveness subscale and a 6-item self-certainty subscale.

A composite index of the BCIS reflecting cognitive insight was calculated by subtracting the score for the self-certainty scale from that of the self-reflectiveness scale.

Drug Attitude Inventory (DAI-10): The aim of this questionnaire to gain some understanding of what people think about medications and what experiences people have of them. The scale has 10 items, 6 of which would be endorsed as True and 4 as false in a non-dysphoric or compliant profile. A 'compliant' response is scored as +1 ; a dysphoric response is scored as -1 . A positive sum of items indicates a positive subjective response (compliant response); a negative sum of scores indicates a negative SR (non-compliant response).

Schedule for Assessment of Insight (SAI; David, 1990) is a semi structured interview assessing three dimensions of insight (treatment adherence, awareness of illness and relabeling of psychotic phenomena).

Berg's Card Sorting Test (BCST) a PEBL version of the WCST (Wsconsin Card Sorting Test), is a measure of prefrontal executive functioning, the task requires problem solving strategies in addition to working memory components.

Conner's Continuous Performance Task (CCPT) measures a person's sustained and selective attention and impulsivity, it' is a vigilance task, which takes approximately 14 minutes to complete.

\section{Analyze}

Collection and statistical analysis of data was realized using SPSS software. Correlational analyses among neurocognition, insight and nonadherence were performed using Pearson correlation coefficients. This statistical analysis was performed using the SPSS version 17.0 software package. Statistical significance was defined as $\mathrm{p}<0.05$.

\section{Results}

$\mathrm{N}=41$ patients

$>$ Gender: $12,2 \%$ female and 87,8 \% male

$\rightarrow$ Mean age: $33,51(+/-9,37)$; with a range of $21-66$.

$\checkmark$ Educational attainment: $0 \%$ unschooled; 26,8 \% elementary; 24,4 \% college; 14,6 \% high school; 34,1 university (Figure 1).

$\rightarrow$ Marital status: 82,9 single; 12,2 married; 4,9 divorced; $0 \%$ widow

$\rightarrow$ Current profession: $61 \%$ no; $39 \%$ yes

$\checkmark$ Family antecedents: $39 \%$ yes

$\checkmark$ Substances misuse: $29,3 \%$ no; $48,8 \%$ cannabis; $26,8 \%$ alcohol; $9,8 \%$ psycho-active medicines; $7,3 \%$ other substances

$\rightarrow$ Mean age of the onset of the disorder: $24,61(+/-6,26)$ with a range of $16-42$.

$\rightarrow$ Mean of the number of hospitalizations: 3,63 Between 1 and 16 times.

D Treatment: 51,2\% Haloperidol; 85,4\% Levopromazine; 31,7\% Chlorpromazine; 9,8 \% Olanzapine; 17,1\% Risperidone; 24,4\% Long acting antipsychotics; 31,7\% Others (Figure 2). 


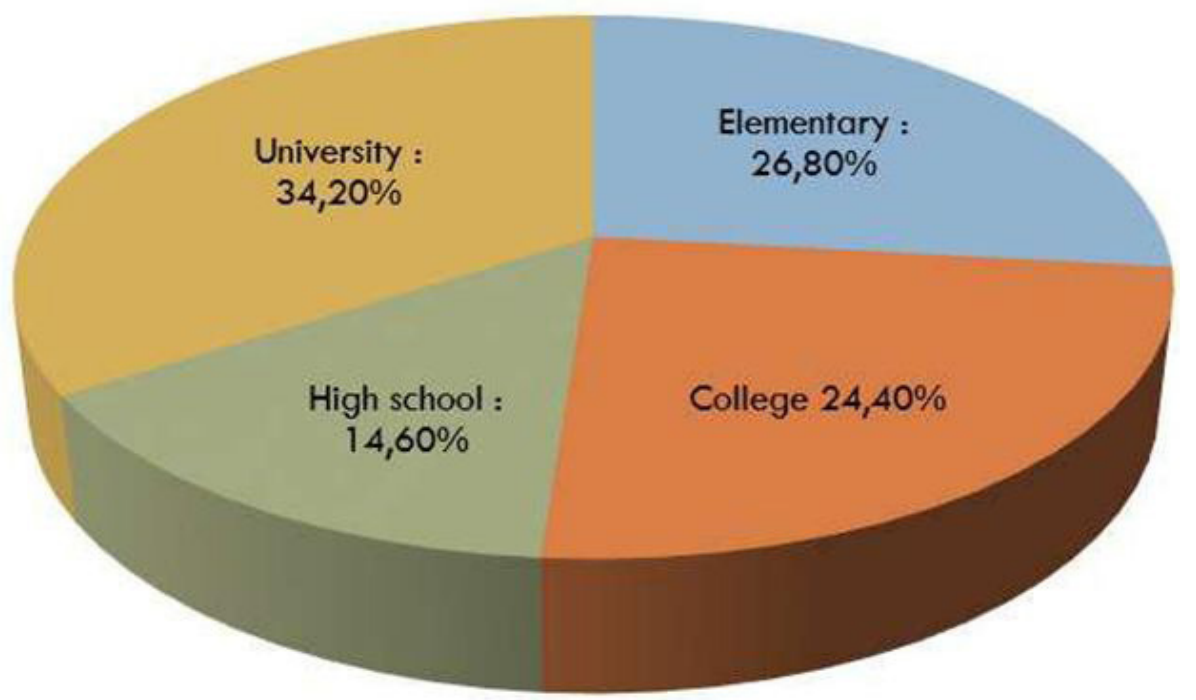

Figure 1: Distribution of patients by educational level

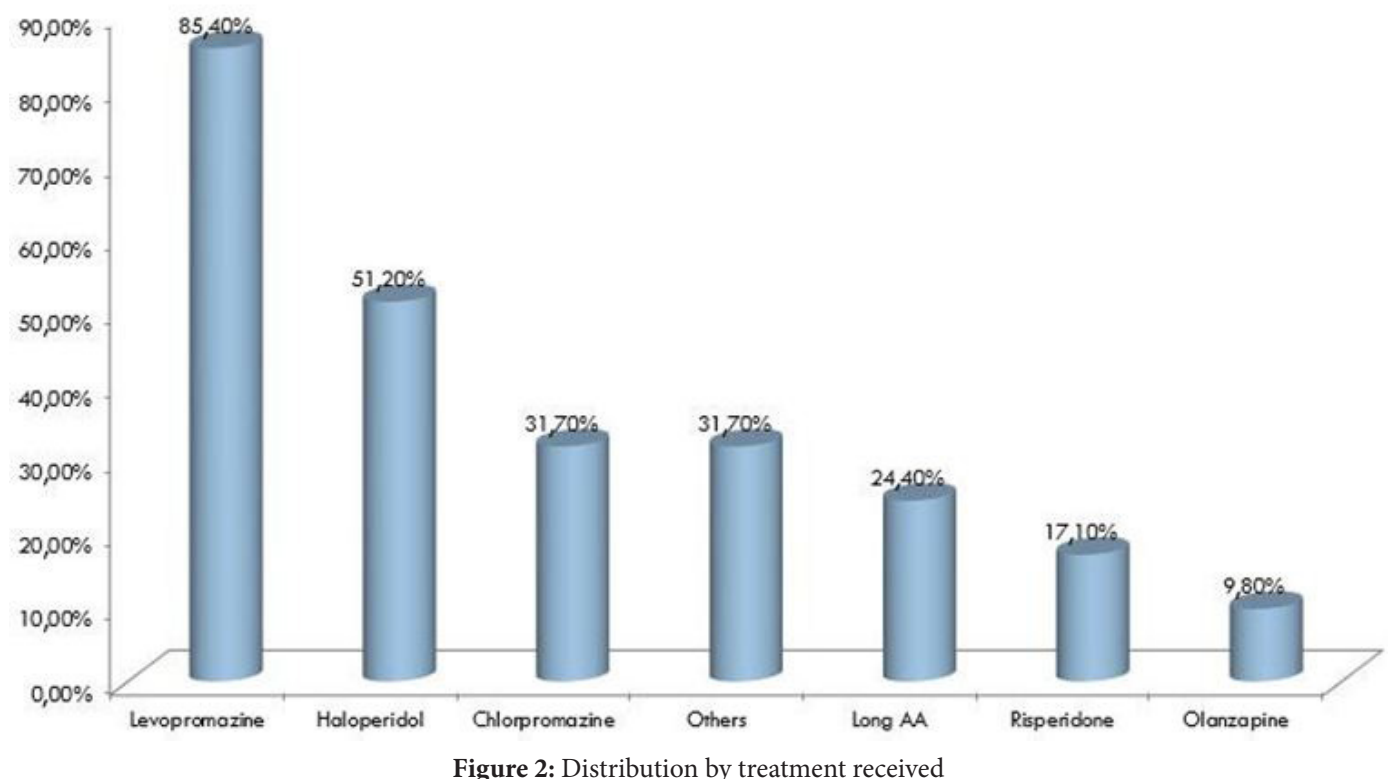

- Statistically significant correlations between: SAI treatment adherence and DAI $(\mathrm{p}=0,001)$, and between SAI relabeling of psychotic phenomenon and DAI ( $\mathrm{p}=0.005)$.

- No statistically significant correlation between: SAI awareness of illness subscale and DAI $(\mathrm{p}=0,4)$.

- SAI awareness of illness subscale statistically correlated with BCIS self-reflectiveness subscale ( $\mathrm{p}=0,007)$ and BCIS composite index subscale $(\mathrm{p}=0,006)$.

- PCPT correct response trial mean inversely correlated with BCIS self-certainty subscale $(\mathrm{p}=0,025)$.

- No statistically significant correlations between: neuropsychological tests (BCST, PCPT) and clinical insight (SAI).

\section{Discussion}

Insight can be broadly classified into clinical and cognitive. The former is a simpler definition which empowers the clinician to understand whether a patient is aware of the symptoms. The later refers to a higher and complex concept of metacognition, which deals with one's ability to examine distorted cognitive views and revise them [5].

The relationship found between clinical insight and attitudes toward taking medication confirmed previous studies [6,7]; but not all the facets of insight were associated with treatment compliance in this study; one explanation is that reasons for nonadherence are multifactorial (current or past drug-dependency, poor relationship with the therapist, shorter length of illness, hospital discharge without an adequate follow-up plan or environment, distress associated with specific side effects or general fear of side effects, inadequate efficacy with persistent symptoms, believing that medication is no longer needed ... [8]); therefore, multiple approaches, beyond the problem of insight, are necessary to address the complex problem of nonadherence [4]. 
The Beck Cognitive Insight Scale has two distinct subscales, self-certainty and self-reflection. Poor insight is characterised by a high degree of certainty in one's (mis)interpretations, and a lack of self-reflectiveness [9].

The relationship between insight of illness and cognitive insight has been investigated by Beck et al. and Pedrelli et al., and a significant positive association was found between self-reflectiveness and clinical insight, whereas no association was found between self-certainty and clinical insight. Thus, the finding in the current study is congruent with the finding in the studies cited above [9].

The inverse correlation between the BCIS self-certainty subscale and the PCPT mean, suggest that the overconfidence in the validity of the patients beliefs could be a predictor of their cognitive impairement.

But although numerous studies report unawareness of illness to be associated with dysfunction on several neuropsychological tests, the findings are inconsistent. For example, although an association of insight with performance on the Wisconsin Card Sorting Test (WCST) has repeatedly been reported, a number of studies have failed to replicate such a relationship [10], which is challenging the hypothesis that insight deficits are exclusively or primarily related to frontal lobe brain dysfunction, at least as measured by the Wisconsin Card Sorting Test.

\section{Limitations and perspectives}

There are several limitations to this study.

Sample size was small; especially the number of females was very small in comparison to the number of males; patients were mostly middle-aged males with mild disease severity and more than 5 years of illness duration. Confirmation is therefore required on more diverse and larger groups of patients.

Our findings should be confirmed in future studies by specifically assessing metacognitive capacities and testing the links with neurocognition, insight and nonadherence.

\section{Conclusion}

As some studies suggest the correlation between clinical insight and cognition, some cognitive therapies could be useful to improve the insight wich is considered as one of the most important factors of treatment adherence in patients with schizophrenia; but it's important to consider the multifactoriel character of insight itself. Thus, further studies may determinate the most influential factors on the insight and how to deal with them.

\section{References}

1. Mingrone C, Rocca P, Castagna F, Montemagni C, Sigaudo M, et al. (2013) Insight in stable schizophrenia: relations with psychopathology and cognition. Compr Psychiatry 54: 484-92.

2. Choudhury S, Khess CRJ, Bhattacharyya R, Sanyal D (2009) Insight in Schizophrenia and its Association with Executive Functions Indian. J Psychol Med 31: 71-6.

3. Boyer L, Cermolacce M, Dassa D, Fernandez J, Boucekine M, et al. (2012) Florence Vaillant Neurocognition, Insight and Medication Nonadherence in Schizophrenia: A Structural Equation Modeling Approche. PLoS One 7: e47655.

4. Stratton J, Yanos PT, Lysaker P (2013) Insight, Neurocognition, and Schizophrenia: Predictive Value of the Wisconsin Card Sorting Test. Schizophr Res Treatment 2013: 696125 .

5. Ćurčić-Blake B, van der Meer L, Pijnenborg GH, David AS, Aleman A (2015) Insight and psychosis: Functional and anatomical brain connectivity and selfreflection in Schizophrenia. Hum Brain Mapp 36: 4859-68.

6. Rocca P, Crivelli B, Marino F, Mongini T, Portaleone F, et al. (2008) Correlations of attitudes toward antipsychotic drugs with insight and objective psychopathology in schizophrenia. Compr Psychiatry 49: 170-6.

7. Beck EM, Cavelti M, Kvrgic S, Kleim B, Vauth R (2011) Are we addressing the 'right stuff' to enhance adherence in schizophrenia? Understanding the role of insight and attitudes towards medication. Schizophr Res 132: 42-9.

8. Acosta FJ, Hernández JL, Pereira J, Herrera J, Rodríguez CJ (2012) Medication adherence in schizophrenia. World J Psychiatry 2: 74-82.

9. Cooke MA, Peters ER, Fannon D, Aasen I, Kuipers E, et al. (2010) Cognitive insight in psychosis: The relationship between self-certainty and self-reflection dimensions and neuropsychological measures. Psychiatry Res 178: 284-9.

10. Aleman A, Agrawal N, Morgan KD, David AS (2006) Insight in psychosis and neuropsychological function: Meta-analysis. Br J Psychiatry 189: 204-12. 


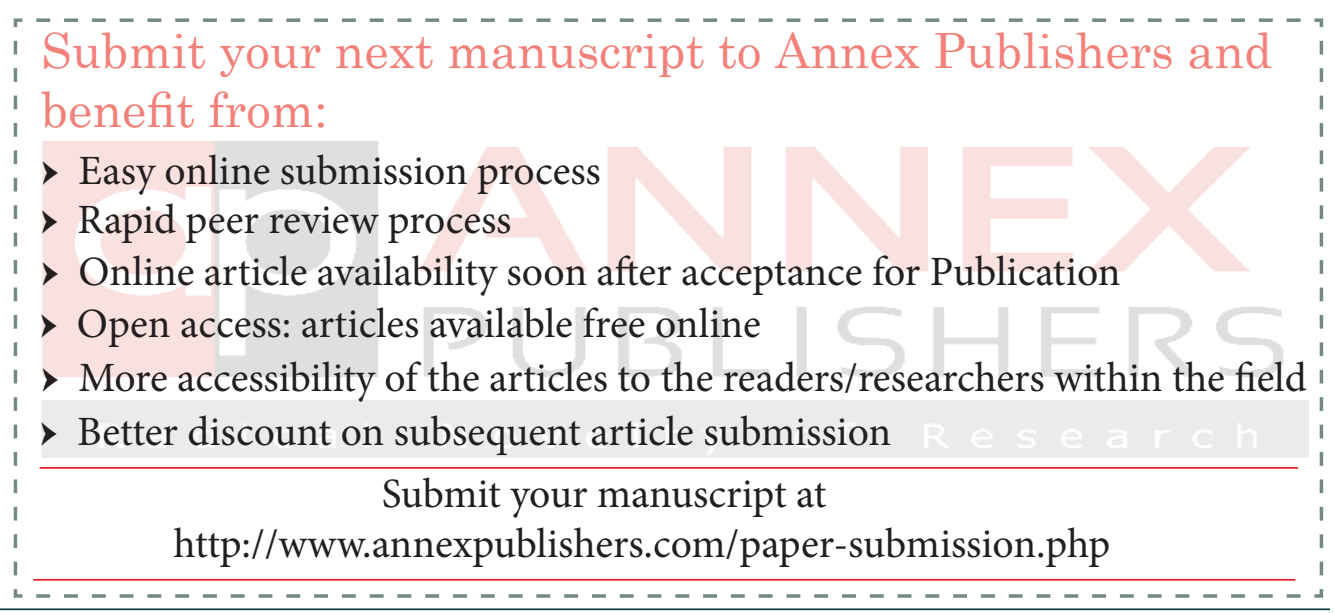

